\title{
Los lenguajes documentales ante el paso de la organización de la realidad y el saber a la organización del conocimiento
}

\author{
Miguel Angel Esteban Navarro \\ Area de Biblioteconomía y Documentación \\ Universidad de Zaragoza \\ 50.009 Zaragoza
}

«Porque los métodos de observación y cooperación han producido incesante progreso y la internacionalización de la ciencia ha ampliado el campo de acción de los investigadores, una clasificación rigurosamente científica ha llegado a convertirse en cosa necesaria» (Paul Otlet y Henri Lafontaine, 1894).

\subsection{Resumen}

Se defiende la hipótesis de que el estudio de la historia del concepto y la tipología de los lenguajes documentales es necesario para construir un concepto de organización del conocimiento preciso y operativo desde una perspectiva documental. Tras discutir el carácter que presenta, se propone la necesidad de ensayar un análisis que atienda a la evolución de cuatro fenómenos: los centros de información y el contenido de los documentos, la concepción del mundo de los documentalistas, los sistemas de clasificación de los científicos y los conceptos de ordenar, clasificar y organizar. La adopción de una perspectiva interdisciplinar permite descubrir la utilidad del estudio histórico de la actividad de clasificación y del análisis de los sistemas, criterios y métodos de construcción de las clasificaciones, efectuados por una rama de la Epistemología: la Taxonomía. La historia del sistema de las ciencias desde Aristóteles hasta el siglo XX y la comparación de las semejanzas y diferencias entre las clasificaciones bibliográficas universales y las taxonomías cientíticas muestra que las primeras asumieron varios de los principales caracteres de éstas durante el siglo XIX, si bien su nacimiento también supuso un cambio revolucionario: el paso de la clasificación de los objetos, las ideas y los saberes a la clasificación de los conocimientos. 
Asimismo, su aparición resolvió algunos problemas epistemológicos planteados por esas clasificaciones en el siglo pasado. Sin embargo, el origen de los dos tipos principales de estructura de los lenguajes documentales, la jerárquica y la combinatoria, debe buscarse en la tradición bibliotecaria. Finalmente, se deducen fórmulas para la optimización de los instrumentos de representación y organización del conocimiento. La artificialidad y modificabilidad de todas las clasificaciones, con su consiguiente relatividad y precariedad, obligan a crear unas clasificaciones documentales flexibles, adaptados a su entorno y con capacidad de integración de los cambios que se producen en la realidad a organizar. (Autor)

Palabras clave: Lenguajes Documentales. Historia. Organización del Conocimiento.

\subsection{Abstract}

The hypothesis about the need of the study of the history and typology of documentary languages is emphasized to elaborate an operative and precise concept of knowledge organization for information retrieval. The actual character of that history is discussed. Alternatively, is proposed an analysis based on the evolution of these facts: the information centers and the content of their documents, the thinking of the information managers, the scientific classification systems and the ideas of order, classification and organization. The adoption of an interdisciplinary approach permits discover the utility of the historical study of the classification activity and of the analysis of the systems, criteria and methods of the classification construction, effectuates by a branch of the Epistemology: the Taxonomy. The history of the system of sciences from Aristote to the 20th century and the comparison of the similitudes and differences among the bibliographic universal classifications and the scientific taxonomies show that the firsts assumed something of the principal characters of these in the 19th century, but their birth implied too a revolutionary change: the passage of the object, idea and thought classification to the knowledge classification. So, their growing resolved something epistemologic problems posed by the scientific taxonomies in the last century. However, the origin of the hierachical and associative structure of the documentary languages must to find in the tradition of librarian work. Finally, techniques are deduced for to optimize the knowledge representation and organization devices. The artificiality and the modificability of all classifications, whit their consequent relative and precarious character, oblige to create some flexible and adaptive documental classifications, whit capacity for to integrate the changes on the nature to organize. (Author)

Keywords: Documentary languages. History. Evolution. Knowledge Organization.

Scire. 1 : 2 (jul.-dic. 1995). 


\section{Necesidad de una aproximación interdisciplinar a la historia de los lenguajes documentales}

La presentación de los lenguajes documentales como sistemas de clasificación y de ordenación suele ser un elemento central de la conceptualización de este instrumento de la representación y organización del conocimiento. Sin embargo, el estudio del carácter y del nacimiento y evolución del concepto de lenguaje documental y de los instrumentos adscritos a éste, se encuentra dominado por una perspectiva poco preocupada por exponer su relación con otras herramientas de organización de la realidad ideadas por el hombre, así como poco interesada por analizar los fundamentos epistemológicos, cognitivos, sociológicos y pragmáticos sobre los que se apoya la construcción y consolidación de su estructura y su variada tipología.

La historia de los lenguajes documentales consiste, generalmente, en la narración acrítica de su convivencia y posterior sustitución de los sistemas de clasificación filosóficos y científicos, la presentación de una colección de definiciones, la crónica o incluso simple catálogo irreflexivo de los hitos de los diversos sistemas construidos hasta la fecha - sin mostrar su conexión con otros fenómenos contemporáneos de las Ciencias de la Documentación o ajenos a éstas-y la descripción de su modo de uso y de las variadas divisiones del Universo que proponen. En definitiva, se trata de una historia que transcurre desde la supuesta primera clasificación documental — la de Calímaco para la biblioteca de Alejandría en el siglo III a. C. - hasta los actuales tesauros y lenguajes de estructura sintáctica, sin apenas tejer otras relaciones entre los diversos tipos de lenguajes documentales y entre éstos y otro tipo de clasificaciones propuestas por el hombre, que la exposición de las relaciones genéticas que guardan unas clasificaciones con otras, construyendo líneas de filiación y paternidad.

Así, el análisis de la relación entre los lenguajes documentales y las taxonomías científicas, suele limitarse a un ingenuo razonamiento circular, caracterizado por la idea de que el nacimiento de las clasificaciones documentales universales supone la sustitución lógica e históricamente necesaria de las clasificaciones científicas por un sistema de ordenación del conocimiento humano más estructurado y perfecto, que se explica por el hecho de que su destino consiste en permitir una mejor organización en un centro de información de los documentos que contienen ese conocimiento. Siendo provocada esa sustitución, simultáneamente, tanto por la creciente complejidad y especialización de los saberes y los trabajos humanos, como por el aumento de los documentos que informan sobre esos saberes y técnicas. Para presentarse, en consecuencia, tales fenómenos como los únicos motores de la evolución de los sistemas de organización documental hasta nuestros días.

Scire. $1: 2$ (jul.-dic. 1995). 
De este modo se ha construido un conjunto de verdades a medias, no tanto porque lo anterior no sea cierto, sino por el hecho de que se ha marginado el estudio de otros hechos tan o más importantes en la explicación del porqué y el modo en que se produjo esa sustitución de las clasificaciones científicas por las documentales. En concreto, qué clasificaciones, sistemas y métodos de ordenar la realidad han influido o están influyendo en la construcción de los sistemas de organización documental. Por ejemplo, no se ha reflexionado por qué razón la estructura jerárquica de las primeras clasificaciones documentales es trasunto fiel de la estructura de las primeras taxonomías científicas empleadas en el siglo XVIII para ordenar el conocimiento que se tenía de las plantas y los animales pensemos en Linneo- - cuando en la época en que surgieron, a finales del siglo XIX y principios del siglo XX, este modelo taxonómico estaba sufriendo una profunda crisis dentro de las ciencias naturales.

Asimismo, al presentar la clasificación documental como un salto cualitativo frente a las clasificaciones científicas, se ha considerado, erróneamente, que los teóricos de la ciencia han dejado de hacer clasificacion; o bien, cuando ésto no se ha ignorado, se ha pensado que tales reflexiones taxonómicas no podían tener ninguna influencia en la evolución y diseño de unos sistemas de clasificación, como los documentales, considerados superiores. Por tanto, al eludir el hecho de que los científicos han seguido haciendo taxonomías e interrogándose sobre la relación entre las ciencias y los saberes, no se ha estudiado cómo las investigaciones taxonómicas han afectado a lo largo del siglo XX al desarrollo de las clasificaciones documentales. Y tampoco se ha investigado cómo han influido los modos documentales de clasificar, tanto en la ordenación de las ciencias efectuada fuera del ámbito de los profesionales y científicos de la documentación, como en los sistemas usados por parte de cada disciplina científica para acceder a la comprensión ordenada de la parcela de la realidad de la que se ocupa.

En definitiva, el dominio de la perspectiva descrita impide disponer de elementos de juicio suficientes para analizar y comprender el cómo y el porqué del estado actual de los rasgos, elementos, estructuras y modos de uso de los diversos tipos de lenguajes documentales; obstaculizando, por tanto, el bosquejo de un marco correcto sobre el que diseñar la adaptación de su naturaleza para responder a los retos que el presente y el futuro puedan plantearle. Porque si el análisis histórico de la evolución de los lenguajes documentales no se efectua con unos presupuestos interdisciplinares, difícilmente se buscarán ideas en otras ciencias para permitir su continua y necesaria recomposición para seguir siendo la principal y más efectiva herramienta de la organización del conocimiento.

Pero la superación de las limitaciones impuestas por la perspectiva descrita no exige sólo una ampliación de los campos de estudio, sino también una reconsideración del método seguido. Se debe sustituir la narración descriptiva y acríti-

Scire. $1: 2$ (jul.-dic. 1995). 
ca del pasado por el ejercicio del verdadero método histórico, donde el conocimiento del pasado sirve para comprender el presente y modelar el futuro, a partir del planteamiento de todos los interrogantes críticos necesarios para desvelar el mayor número de relaciones que concurren para explicar el porqué de los hechos.

El estudio de la formación y evolución de los sistemas de representación y organización documental del conocimiento desarrollados en los últimos cien años, debe atender, fundamentalmente, al análisis y comprensión de la influencia que han tenido y tienen cuatro fenómenos, cuyo resultado definitivo no podemos mostrar, ya que todavía nos encontramos inmersos en su estudio. En primer lugar, la variación de la cantidad, calidad y tipología de la información y los documentos generados por el hombre, y, en correspondencia con aquélla, la evolución de los centros dedicados a su conservación y difusión. En segundo lugar, la valoración que tanto los teóricos de la documentación y de sus instrumentos, como los distintos sectores profesionales dedicados a la gestión de la información, han efectuado de la utilidad de los diversos tipos de lenguajes documentales para hacer frente con éxito a las necesidades planteadas por la aparición de nuevas realidades documentales y el cambio en las demandas de los usuarios; incidiendo, sobre todo, en el análisis de la tradición cultural, la concepción de las necesidades de los usuarios, el modelo de centro de información y la sociología profesional de esos pensadores y grupos. En tercer lugar, el efecto del desarrollo de los sistemas científicos de clasificar y ordenar la realidad; atendiendo, en especial, a los criterios que guían la división de la realidad, la agrupación de los elementos en clases y la construcción de su estructura, ya que tras toda clasificación documental existe una asunción más o menos consciente por su elaborador y usuarios del sistema de conocimiento de la realidad dominante en su tiempo. Y, en cuarto lugar, la evolución en el ámbito de las ciencias cognitivas de la concepción de ordenar, clasificar y organizar la realidad por la mente humana, con objeto de establecer el mapa de aportaciones e intercambios entre los diversos paradigmas epistemológicos y los sistemas de organización documental.

\section{Contribuciones de la Taxonomía para la construcción de una teoría de la Organización del Conocimiento}

La Botánica y la Zoología fueron las primeras ciencias que desde el Renacimiento se ocuparon de la confección de clasificaciones como un medio de estudio de la realidad, procediendo a la agrupación y organización de las plantas y de los animales mediante la construcción de unos sistemas jerárquicos de categorías basados en las relaciones naturales presumidas entre los organismos, que se conocen como taxonomías. Estos trabajos clasificatorios tuvieron tanta importancia en el desarrollo del conjunto de las ciencias naturales, que adquirieron incluso el rango de una especialidad independiente dentro de éstas.

Scire. 1 : 2 (jul.-dic. 1995). 
Sin embargo, filósofos, psicólogos, lingüistas, antropólogos, sociólogos, etnólogos, físicos, químicos... también deben realizar, en mayor o menor medida, una clasificación de la realidad que analizan, para lograr la plena comprensión de sus fenómenos. La mayoría de la ciencias necesitan construir en algún momento de su evolución, haciendo uso del método comparativo, una clasificación de los elementos de la parcela de la naturaleza física o social de la que se ocupan, que les permita efectuar una presentación estructural o sistémica de su objeto de estudio. Por consiguiente, aunque, en atención a su origen, existe una concepción reducida de taxonomía como «clasificación biológica», su significado etimológico se halla más acorde con la realidad: ley o manera acerca de cómo deben ser dispuestos los objetos o hechos, ya que procede de la combinación de los términos griegos taxis (clase, disposición) y nomos (ley, norma, regla).

En definitiva, conviene destacar que el análisis del proceso de clasificación y el diseño de los instrumentos más adecuados para llevar a cabo esa operación, no interesan exclusivamente a las Ciencias de la Documentación, sino que consisten en unas tareas que ocupan un lugar central en el trabajo del resto de las ciencias. Así, no ha de sorprender el desarrollo durante nuestro siglo de una nueva disciplina: la Taxonomía o «ciencia de la clasificación», como una rama de la Epistemología o Teoría de la Ciencia dedicada fundamentalmente a dos actividades. Por un lado, al estudio de la evolución de las clasificaciones de los saberes y de los productos del conocimiento científico realizados a lo largo del tiempo. Y, por otro, al análisis de los sistemas, criterios y métodos de construcción de las clasificaciones científicas, atendiendo a la tipología, propiedades y peculiaredades de los objetos o ideas a clasificar.

La historia de los sistemas científicos y la construcción de nuevas clasificaciones de las ciencias que mantengan la presentación de las diversas disciplinas en grupos semiautónomos, pueden parecer unas labores inútiles para responder a las necesidades del presente, ya que sugieren una impresión de aislamiento que no casa con la actual tendencia a la integración del sistema de las ciencias a partir de la unificación del método científico. Sin embargo, ambas tareas son de gran utilidad para deducir fórmulas de optimización de este método, ya que, por un lado, permiten detectar las variaciones y tendencias en la definición de los campos singulares de acción de cada disciplina o grupo de ciencias, y, por otro, sugieren líneas de aproximación entre ellas, capaces de permitir un fructífero intercambio de técnicas y de resultados de la investigación. En definitiva, nos invitan a sugerir fórmulas de transmisión del conocimiento más fecundas dentro de una misma disciplina y entre las diversas disciplinas, que tengan en cuenta la continua movilidad del mapa de las ciencias, presidida en la actualidad por el principio de la integración y la tendencia a la transdisciplinariedad, con objeto de superar la crisis producida por la quiebra del sistema monodisciplinar, caracterizado

Scire. $1: 2$ (jul.-dic. 1995). 
por el aislamiento y la parcelación del saber provocados por el espectacular aumento y especialización de las técnicas de investigación en los dos últimos siglos.

Por otra parte, ni los científicos han dejado de clasificar ni los espistemólogos han renunciado a presentar clasificaciones del saber, porque hayan surgido clasificaciones documentales. Hoy se siguen efectuando taxonomías, y una más o menos consciente valoración o perspectiva taxonómica de la información creada, se encuentra en la base de todo trabajo intelectual. Todo científico y toda conquista de la ciencia se situa y se produce en el seno de una disciplina científica, que a su vez se integra de un modo peculiar con el resto de las disciplinas en el marco general de la ciencia, definiendo una serie de relaciones - fundamentalmente de integración, superioridad, subordinación o complementariedad—, que rigen y modelan unos fenómenos cuyo conocimiento es necesario tanto para el constructor de clasificaciones documentales como para el documentalista. En concreto, el grado de consolidación e influencia de una disciplina en la comunidad científica; la formación y justificación de los equipos científicos; los modos y vías de difusión, transferencia e intercambio de datos, métodos y tesis; la aceptación de determinadas teorías, con la correspondiente obtención de elementos y estrategias para la elaboración de hipótesis; el uso de instrumentos y técnicas de observación y de análisis; y la utilización de una terminología y unos mapas conceptuales determinados. Por consiguiente, conocer cómo se han constituido y evolucionan las taxonomías y los mapas de la ciencia vigentes en una determinada comunidad científica, podrá ser para el documentalista un eficaz auxilio para proceder a la organización de los documentos que aquélla genere.

\section{Artificialidad, modificabilidad, relatividad y precariedad de las clasificaciones}

La principal aportación de esta necesaria vertiente historicista de la Taxonomía a la geografía de la ciencia y, por ende, también a la Organización del Conocimiento, consiste básicamente en el descubrimiento de la artificialidad y modificabilidad tanto de los sistemas de la ciencia como de las clasificaciones de la realidad; de lo que resulta, la afirmación de la relatividad y la precariedad de ambos.

La Taxonomía nos enseña que no existe un sistema de clasificación esencial, natural o único que sea adecuado a priori a la naturaleza de la realidad. La confección de una clasificación obedece, en última instancia, al uso por los científicos y los filósofos de la ciencia de unos criterios intelectuales condicionados por los rasgos generales de su tiempo, y, en particular, por el estado en el que se encuentra el desarrollo de los recursos epistémicos; y sólo aparentemente al supuesto carácter de una realidad objetiva situada en una naturaleza inmutable, a

Scire. $1: 2$ (jul.-dic. 1995). 
pesar de que la mayoría de las taxonomías se nos intentan presentar como las más adecuadas a esa supuesta realidad objetiva. Como destaca Vickery : «La historia se compone de una serie de épocas culturales. En un mismo período de años, dentro del cual el conocimiento presenta una más o menos unificada estructura, éste puede expresarse en una clasificación; pero cada nueva época requiere una nueva clasificación» (Vickery, 1975, p. 147). Cada época tiene su propio carácter y mantiene un especial modo de pensar, que sirve de armazón de sus teorías; es decir, cada período histórico de la ciencia estudia unos problemas muy particulares, que obedecen a unos intereses muy singulares, con unos métodos de estudio peculiares.

Por consiguiente, podemos deducir el siguiente corolario: «cuánto más se adapta una clasificación a una época determinada, menos apropiada será para otra época» (Vickery, 1975, p. 147). En consecuencia, no ha lugar juzgar en abstracto si una clasificación es mejor que otra, sino que se debe atender al análisis del propósito para el que se ha construido, y ésto en el marco de su contexto histórico. La validez, mejor la pertinencia, de toda clasificación de la ciencia debe ser siempre relativizada, ya que éstas varían según aumenta la cantidad y la calidad del conocimiento científico y se modifica la concepción que los expertos tienen de la ciencia. Toda taxonomía científica es un producto histórico, y, por tanto, se encuentra siempre inmersa en un proceso de cambio, que origina nuevas formas para sustituir a las agotadas por el paso del tiempo. Como máximo, podemos mantener que un sistema clasificatorio es superior a otro porque puede aplicarse en un número mayor de contextos culturales. Asimismo, cuanto más se adecue una clasificación al orden conocido y consensuado de la naturaleza en un momento histórico dado, más útil y práctica será.

En definitiva, un rasgo común de todas las clasificaciones, todavía más acusado en las actuales, es su caducidad, si no precariedad, debido a que las ciencias están en continua formación, a que ciertos territorios límites dan lugar con frecuencia a ciencias nuevas, a que la evolución de los paradigmas, teorías, métodos e incluso los objetos de estudio cambian, continuamente, la imagen de la realidad y provocan la redefinición de las relaciones entre las ciencias. Por consiguiente, son inútiles, por no tener en cuenta este hecho, todos los esfuerzos taxonómicos que se presentan como una completa y definitiva integración de todo el conocimiento humano bajo un número determinado y definitivo de etiquetas y cabeceras. Ningún sistema puede pretender clasificar completamente ni la realidad ni el conocimiento en un estado dado de su desarrollo. Por consiguiente, el estudio de los sistemas de las ciencias y de las taxonomías de la naturaleza debe tener en cuenta que éstas evolucionan al mismo ritmo que la propia humanidad. Ritmo que en la actualidad es desenfrenado, debido a que los avances del conocimiento científico y los nuevos descubrimientos e inventos nos invitan, con una frecuen-

Scire. $1: 2$ (jul.-dic. 1995). 
cia cada vez mayor, a modificar nuestros paradigmas o concepciones del mundo. Así, si bien la clasificación aristotélica del saber estuvo vigente durante casi dos mil años, los sistemas de las ciencia que se han propuesto desde el Renacimiento, y sobre todo desde el siglo XIX, se agotan con una rapidez inusitada. No obstante, esta precariedad no significa que las taxonomías científicas hayan sido o sean inútiles, ya que representan un profundo esfuerzo por sistematizar y ordenar cuerpos dispersos de conocimientos.

Estas conclusiones que se deducen del análisis histórico de las clasificaciones humanas, se pueden aplicar también a los sistemas de organización documental, ya que sólo se tratan de una especie de ese género mayor, y, por tanto, nos sirven para idear fórmulas capaces de optimizar mejor su gestión y sus funciones. En concreto, nos obligan a definir, construir y mantener sistemas de clasificación singulares, adaptados y flexibles para cada disciplina o grupo de disciplinas y de acuerdo a diversos contextos; es decir, con capacidad de integrar los cambios que resultan de la variación del estado disciplinar de cada ciencia y de las mutaciones que experimenta en la organización de los fenómenos investigados. La gestión de clasificaciones universales del saber es una misión imposible, no tanto por la envergadura de la empresa, como por el ciego empeño de sus defensores por nadar contra corriente, estancándose en un doble anacronismo, pues no sólo se proponen trazar un imposible mapa general de las ciencias, sino que aspiran, incluso, a representar la imagen taxonómica que cada disciplina tiene de su campo de estudio, con la ingenua convicción de que sus variaciones son los pasos que se dirigen, de modo inevitable, a la cumbre donde habita un reflejo exacto del orden del Universo.

\section{De las clasificaciones de las ciencias a las clasificaciones docu- mentales}

Como complemento de ese interés pragmático, el análisis de la evolución de la clasificación también nos permite detectar, desde un punto de vista exclusivamente histórico, el contexto en el que surgieron los primeros lenguajes documentales, los elementos que tomaron prestados de las clasificaciones de las ciencias y la revolución que su nacimiento supuso tanto para la técnica de la clasificación como para la gestión de los documentos.

La comprensión de esos tres fenómenos exige esbozar, previamente, los rasgos de las clasificaciones propuestas desde la Antigüedad clásica hasta nuestros días, con especial atención a las realizadas a partir del Renacimiento. Evidentemente, la clasificación de las ciencias es un tema específicamente moderno, pues sólo apareció con el reconocimiento de la independencia de las ciencias particulares con respecto a la filosofía. Sin embargo, en un sentido amplio, podemos identificar la clasificación de las ciencias con la clasificación de

Scire. $1: 2$ (jul.-dic. 1995). 
los saberes, y, por tanto, comprender dentro de ella las subdivisiones de la filosofía propuestas por los filósofos clásicos y medievales.

Asimismo, consideramos que tal estudio debe basarse en la asunción de tres presupuestos básicos. Primero, que la clasificación de las ciencias se trata de un problema filosófico, pues en todo ensayo se encuentra implicada una concepción del saber y, por tanto, de las relaciones que se establecen entre los diversos saberes científicos y entre éstos y los conocimientos no científicos. Segundo, que los motores de su evolución se encuentran, por igual, en el concepto de ciencia dominante en la comunidad científica de cada época, en la percepción por ésta de la cantidad y calidad del conocimiento generado y en las diversas situaciones sociales e ideológicas de los gremios protagonistas de cada saber. Y, tercero, que se deduce de lo anterior, que la pertinencia de toda clasificación de los ámbitos del saber y de las disciplinas científicas ha de ser siempre relativizada desde una perspectiva histórica y cultural.

La clasificación del saber o de la ciencia propuesta por Aristóteles - pues utilizó indistintamente los términos episteme y philosophia para referirse a la misma realidad-, gozó durante muchos siglos de gran prestigio e influencia. El Estagirita clasificó los "saberes" en tres grupos, en función de sus diversos objetos: teóricos, prácticos y creativos. Los saberes teóricos (Theoria) se ocupan del análisis de la verdad de las cosas reales: las ideas, con sus formas y sustancias. Se basan en una forma de conocimiento que se agota en sí mismo: el saber por el mero saber. Están formados por las actuales ciencias formales y las ciencias que hacen uso del método experimental. En cambio, los saberes prácticos (Praxis) se dedican al análisis de las acciones humanas encaminadas a un fin, por lo que persiguen el establecimiento de un saber capaz de guiar la conducta humana. Incluyen, entre otros, la Ética, la Política, la Económica y la Retórica. Y, por último, los saberes poéticos o creadores (Poiesis) son los encaminados a la utilización y transformación técnica (Techné) de las cosas reales para el servicio de la vida humana.

El sistema aristotélico fue el marco de la clasificación del conocimiento humano en las culturas clásica, cristiana y musulmana a lo largo de casi dos mil años: desde el siglo IV a. C. hasta el Renacimiento. Así, la mayoría de las clasificaciones efectuadas por los teólogos y filósofos medievales mantienen una estrecha dependencia del sistema aristotélico, que nos permite deducir una serie de rasgos comunes. La afirmación de la unidad del saber, sin reconocer la existencia de ciencias o saberes particulares, que son considerados únicamente facetas o aspectos de un saber único y universal, debido al dominio del principio de la reductio artium ad theologiam. La asunción del criterio clasificador de fijarse en el objeto. La tendencia a organizar los saberes singulares de modo piramidal, de acuerdo con los grados de abstracción que exigen cada uno de ellos; siendo

Scire. 1 : 2 (jul.-dic. 1995). 
usual presentar la serie: física, matemática y metafísica, respetando, incluso casi sin cambios, las divisiones propuestas por Aristóteles para la física. Y, por último, el tendido de puentes entre la ciencia y unos ámbitos del conocimiento menos abstractos y con una dimensión profundamente técnica, sino artesanal, como era el caso de las denominadas artes mecánicas; cuyos avances acabaron incorporándose en la clasificación, sobre todo a partir del siglo XIII, dándoles una categoría propia junto con los tres saberes propuestos por el esquema aristotélico.

Destacaron, por su difusión, las clasificaciones del saber propuestas por Alfarabi en el Catálogo de las ciencias, en el siglo X; Avicena en su monumental comentario a Aristóteles, La Sabiduría en el siglo XI; el inglés Hugo de San Victor a partir de los textos de Avicena en el siglo XII; Domingo Gundisalvo en De divisiones philosophiae, también en el siglo XII, siguiendo la tradición aristotélica árabe; y el franciscano San Buenaventura en De reductione artium ad theologiam, en el siglo XIII. Junto a estas clasificaciones que seguían un criterio filosófico, se realizaron otras según un criterio pedagógico; siendo la más conocida, la que dividía la enseñanza de las siete artes liberales, que permitía en las Universidades el acceso a los estudios superiores de Teología, enTrivium (Gramática, Dialéctica y Retórica) y Quadrivium (Aritmética, Geometría, Música y Astronomía).

Sin embargo, la dificultad del esqueleto del sistema de clasificación aristotélico para integrar las nuevas ciencias que desde el fín del Medievo nacían como abstracción y guía de las técnicas, junto con el desmembramiento de la concepción unitaria del saber que supuso el nacimiento de la especialización científica durante el Renacimiento, acabaron provocando su crisis. La puntilla la recibió del filósofo y político inglés sir Francis Bacon en 1620, cuando en su obra Instauratio Magna negó que el conocimiento pudiera dividirse en teórico, práctico y productivo, y propuso una clasificación jerárquica basada en la división de las facultades humanas, donde cada una de ellas origina un tipo de saber, que a su vez se divide en una serie de disciplinas subordinadas. La memoria origina la Historia, que se divide en sagrada, civil y natural; de la razón nacen las ciencias de la Naturaleza (Metafísica y Física) y del Hombre (Lógica, Ética y Ciencia de la Sociedad); y de la fantasía o imaginación surgen las Artes. Así, aunque las agrupaciones básicas de la física y la matemática aristotélicas permanecían casi inmutables en el esquema baconiano, cambiaban las razones dadas para agrupar las disciplinas que componían esas materias, y, por consiguiente, se abría la puerta a la proliferación de sistemas de clasificación competitivos con el aristotélico. El cual entró en un declive más pronunciado, hasta su colapso final a comienzos del siglo XVIII, ante la incapacidad para acomodarse al desarrollo y a las nuevas concepciones de las disciplinas científicas clásicas.

Scire. $1: 2$ (jul.-dic. 1995). 
Aunque el sistema de Bacon gozó de gran difusión hasta él siglo XIX, no pudo conseguir un éxito y una universalidad similares a los gozados por el aristotélico. En consecuencia, el reto con el que los filósofos se enfrentaron a partir de la defunción de ese sistema, fue construir y elegir otro capaz de llenar el vacío que había dejado y de integrar los continuos avances científicos. No se abstuvieron de participar en esta empresa los más destacados filósofos y científicos de los siglos XVII y XVIII, aportando diversos criterios clasificadores y reflexionando sobre algunos de los fundamentos del trabajo taxonómico. Entre otros, merecen figurar en esa nómina, Tommaso Campanella con su Ciudad del Sol, en 1623; J. A. Comenius con un opúsculo dedicado a esta materia dentro de su Pansophiae prodromus, publicada en 1639; Thomas Hobbes con el capítulo noveno de Leviatán, en 1651; John Locke con el último capítulo de su obra Ensayo sobre el entendimiento humano, publicada en 1690; G. W. Leibnitz en diversos y dispersos escritos de su amplia obra; John Lowthorp con su enumeración de veintiséis ciencias en su Philosophical Transactions, publicada en 1725 bajo el patrocinio de la Royal Society de Londres; Juan le Rond D'Alembert en el Discurso preliminar de la Enciclopedia (1751); Emmanuel Kant en Crítica de la razón pura en 1781; G. W. F. Hegel en su Lógica en 1812...

De la misma época datan las primeras clasificaciones propuestas por diversos humanistas para construir bibliografías u organizar las bibliotecas que les tocó cuidar. El bibliógrafo alemán Georg Draudius en Bibliotheca Classica (1625), el médico Jean Cécile Frey en la parte cuarta de su obra Via ad divas scientias (1625), el jesuita Claude Clement en Musei sive Bibliothecae tam privatae quam publicae extructio, instructio, cura, usus libri IV (1635), el bibliotecario del Colegio de los Jesuitas de París Jean Garnier en su Systema bibliothecae... (1678), el erúdito Daniel Georg Morhof en el Polyhistor (1687-92), Hans Lomeier en el capítulo XIV de su obra De Bibliothecis liber singularis (1705) o Giusto Fontanini en la Dispositio Catalogi Bibliothecae Iosephi Renati Imperailis... (1709) presentaron las propuestas más interesantes o influyentes. (Serrai 1977, p. 95-216; Kumar, 1989, p. 378-380)

Durante el siglo XIX, el problema de clasificar las disciplinas tradicionales, se amplió con el deseo de hallar un modo de integrar y relacionar con éstas, preferentemente en términos de filiación, las nuevas ciencias y técnicas que surgían como resultado de las revoluciones científica e industrial. La mayoría de los sistemas de clasificación propuestos se realizaron decantándose por una u otra de las dos opciones que presentaban los planteamientos de división y agrupación dominantes en ese tiempo. Respecto a la división, se discutía si era factible la reunión de disciplinas distintas en un mismo conjunto o preferible la alternativa de la separación en grupos opuestos. En cuanto a la agrupación de las ciencias, se podía optar por seguir un modo líneal, construyendo series, o mantener un crite-

Scire. $1: 2$ (jul.-dic. 1995). 
rio de generación de clases que se agrupan en una estructura jerárquica de tipo arbóreo, que permite distinguir entre disciplinas principales o secundarias según su grado de complejidad o dependencia mutua.

El esquema jerárquico arbóreo siguió dominando durante todo el siglo XIX, si bien el monopolio que había disfrutado en los siglos anteriores como sistema de agrupación lógica por excelencia, a partir de la presentación de las reglas aristotélicas de división de los géneros en especies en forma de árbol, efectuada por el filósofo neoplatónico Porfirio en su obra Isagogé en el siglo III a. C., se quebró por la irrupción de un nuevo sistema de agrupación basado en la ordenación por series. Augusto Comte fue su principal impulsor, cuando en la segunda lección de su Curso de Filosofía Positiva (1830-42) agrupó las ciencias en un orden caracterizado por una generalidad o extensión decreciente y una complejidad creciente, estableciendo una jerarquía de acuerdo con su grado de positividad, con una prelación lógica donde cada disciplina depende de los conocimientos de la anterior para construir los suyos, pero no de la posterior, de la que resultó la famosa serie positivista de las ciencias: Matemáticas, Astronomía, Física, Química, Biología y Física Social o Sociología. Herbert Spencer continuó el trabajo del pensador francés, si bien planteó un orden bastante diferente: Lógica, Matemáticas, Mecánica, Física, Química, Astronomía, Geología y Biología. Este sistema de ordenación es bastante limitado, pues aunque expresa el hecho de la dependencia entre las ciencias, no revela cómo se establecen las relaciones entre ellas. De ahí que otros seguidores de este criterio, plantearan un ordenamiento basado en la naturaleza del principal recurso o elemento del intelecto humano puesto en acción para el ejercicio de cada ciencia, que adoptó diversas formas en cada autor. William Whewell hablaba en Filosofía de las ciencias inductivas (1840) de la idea que sustenta la construcción de cada ciencia: espacio, número, sígno, límite, movimiento, fuerza... P. E. Dove prefería usar el término nombre objetual, para referise a esas categorías. Y el químico y Premio Nobel de 1909 Wilhelm Ostwald, distinguía las ciencias por los conceptos centrales en la articulación de su discurso: orden, multiplicidad, cantidad, espacio, movimiento, energía y vida (Vickery, 1975, p. 156-157).

Frente a este enfrentamiento entre agrupación seriada y jerárquica, la aparición en 1876 de la Clasificación Decimal de Melvil Dewey supuso una original combinación de ambas posibilidades, con su presentación de diez árboles jerárquicos, donde cada uno de ellos procedía de un elemento de una serie de igual número, convertido en clase originaria. Sin embargo, la explotación, hasta sus últimas consecuencias, de la potencialidad contenida por el sistema seriado como alternativa a las estructuras arbóreas, sólo llegó con los trabajos de S. R. Ranganathan. El matemático y bibliotecario hindú ha sido el teórico de la organización del conocimiento que mejor ha asumido la crítica implícita en el siste-

Scire. $1: 2$ (jul.-dic. 1995). 
ma seriado al irremediable naufragio de los sistemas jerárquicos, que provoca la necesidad de optar por el Escila de la insuficiencia explicativa, por su obligación lógica de elegir un único aspecto de la realidad para convertirlo en el criterio de división generador de un grado divisional, o por el Caribdis de su precariedad lógica cuando opta por la combinación de diversos y variados criterios clasificadores para aumentar su potencia explicativa de la realidad, a cambio de disminuir su eficacia como sistema clasificador. Las deudas de Ranganathan con el sistema de agrupación seriado que se desarrolló en el siglo XIX son tantas, que incluso las cinco categorías de identificación de clases (tiempo, espacio, energía, materia y personalidad) de su sistema de clasificación facetado, encuentran un precedente directo en los conceptos presentados por Ostwald para ordenar las ciencias.

En cuanto a la congregación o separación de las parcelas del conocimiento y de la acción humana en un único o varios sistemas, dominó, hasta bien entrado nuestro siglo, una preferencia por las dicotomías; es decir, por la no integración de esas parcelas en un mismo conjunto, propiciando su separación y colocación en dos bandos irreconciliables.

La división más habitual fue la que presentaba a las ciencias enfrentadas en puras y aplicadas o especulativas y descriptivas, basándose en el tipo de operación mental que exige una ciencia para producir conocimiento. Adoptó diversas denominaciones según los autores: abstractas y concretas (August Comte y Herbert Spencer), puras y empíricas (Arthur Schopenhauer), puras y mixtas (Coleridge), formales y empíricas (Karl Pearson), fundamentales y dependientes (Alexander Bain), generales y aplicadas (Edmond Globot) o generales y especiales (Thomas Wundt). Estas distinciones ya se encontraban implícitas en las últimas clasificaciones medievales, con su división de las ciencias teoréticas y de las artes mecánicas en distintas clases. Como los seguidores de esta perspectiva dividían y agrupaban las ciencias de acuerdo con criterios subjetivos o distinciones idealistas, sin interesarse por el ámbito material en el que actúan y la realidad objetiva de la que se ocupan cada una de ellas, tampoco existía una plena simetría entre un grupo y otro, de modo que un buen número de disciplinas cambiaban de un sistema a otro su adscripción a una clase determinada. La ausencia de una base firme para proceder a una división tan tajante en el sistema de la ciencias, pues en realidad todas las ciencias tienen una parte abstracta y teórica y otra parte empírica y descriptiva, provocó una amplia desorientación y confusión intelectual, que explica esa proliferación de clasificaciones.

Hasta el último cuarto del siglo XIX no comenzó a variar esta actitud, tras la afirmación por C. W. Shields de que «un esquema filosófico de las ciencias debería basarse sobre los hechos que las soportan, antes que sobre las ideas que las envuelven; de tal modo que reflejaría completamente todas las clases distintas de hechos que han sido científicamente determinados y exhibiría todas las clases de

Scire. $1: 2$ (jul.-dic. 1995). 
hechos en sus actuales conexiones (relaciones)» (cfr. Vickery, 1975, p. 159-160). Este cambio de una posición idealista a otra materialista, abrió el paso a un segundo tipo de clasificación dicotómica de las ciencias, basada en los caracteres peculiares de los hechos que estudia cada disciplina, y, en correspondencia con ellos, los rasgos diferenciales del método de investigación usado.

El resultado más conocido de este criterio fue la distinción en el primer grado divisional entre ciencias físicas o naturales y ciencias normativas o humanas, cuyas formulaciones más radicales negaban incluso el carácter científico del segundo grupo. El principal expositor de esta perspectiva fue el filósofo alemán Wilhelm Dilthey. Su propósito era reivindicar el valor de las disciplinas humanísticas, disminuido ante el prestigio adquirido por los saberes sobre la Naturaleza y las técnicas gracias a sus diarios e impresionantes avances, mediante la elaboración de un concepto particular de ciencia para aquéllas, que sirviera para recuperar una calificación que sus competidoras acaparaban en exclusiva. Así, distinguió entre ciencias de la naturaleza y ciencias del espíritu a partir del hecho de que junto a la pecepción externa de las cosas como fenómenos, existe otro conjunto de percepciones internas sobre el interior del hombre, que constituye el espíritu; y, por consiguiente, a cada una de esas realidades y formas de percibir corresponde un tipo distinto de método: la naturaleza debe explicarse (Erklären), y el espíritu comprenderse (Verstehen). La explicación consiste en la ordenación relacional de una serie de fenómenos percibidos. Y la comprensión es la penetración profunda en la realidad, tal como se manifiesta en el espíritu humano, para descubrir valores; correspondiendo a la hermeneútica su realización.

La propuesta de Dilthey tuvo una gran influencia tanto en el pensamiento científico como en el mundo académico de finales del siglo XIX y principios del siglo XX, y sirvió para difundir un sistema de clasificación de las ciencias que ya había recogido incluso unas décadas antes su famosa distinción, si bien bajo la denominación de ciencias cosmológicas y ciencias noológicas o del pensamiento. Se trata de la clasificación elaborada por el padre de la electrodinámica André Ampère, que se nos presenta como arquetipo de las tendencias dominantes en el siglo XIX, ya que conjuga las perspectivas dicotómica y jerárquica. En primer lugar divide las ciencias en cosmológicas o de la Naturaleza y noológicas o del Pensamiento, que a su vez se dividen en otras dos ciencias y, así, sucesivamente, cada una de ellas, a lo largo de seis grados divisionales hasta sumar un total de ciento vientiocho ciencias. Esta clasificación, junto con la del filosófo norteamericano C. S. Peirce, fue uno de las escasos sistemas que profundizaron en los escalones inferiores, ya que, a pesar de la proliferación de propuestas, muy pocas acometieron una subdivisión detalladas de las ciencias.

Si bien, ya durante el siglo XIX se levantaron algunas voces - como las de Thomas Young, Neil Arnott, J. D. Brown y E. W. Hulme- en contra de esta ten-

Scire. $1: 2$ (jul.-dic. 1995). 
dencia dicotómica y en defensa de la unidad de las ciencias, con unos criterios que encontramos años después en las obras de Bliss y Ranganathan, el dualismo continuó dominando hasta el triunfo de la filosofía neopositivista a mediados del siglo XX. El neopositivismo presentó como sostén de su proyecto de unificación interna de cada una de las grandes disciplinas, como modo de recuperar la perspectiva científica global perdida con la especialización y la dispersión disciplinar, la unidad de las ciencias basada en el empleo por todas ellas del método científico y de un metalenguaje fisicalista. Para proceder, sobre el resultado de ese trabajo, a la construcción de un sistema de las ciencias abierto y evolucionable, basado en la agrupación de las disciplinas en diversas clases, sin matiz valorativo adscrito al lugar que cada una ocupa en el esquema, según su grado de asunción de un modelo ideal de ese lenguaje, identificado con la lógica formal, cuyos fundamentos y procedimientos también fueron reformados.

Desde entonces, el centro de atención de los geógrafos de la ciencia se ha desplazado de la construcción de un único mapa universal de la ciencia, a la elaboración de muchos y variados mapas, mediante dos nuevos tipos de trabajos que proponemos denominar con los siguientes nombres. La "intrageografía", centrada en el bosquejo de los rasgos y las regiones internas de cada disciplina, que condicionan su relación con el resto. Y la "kinegeografía", preocupada por el seguimiento de la variación y movimiento de las fronteras entre las ciencias, a partir del análisis de los fundamentos epistémicos y sociológicos que influyen en el avance, los retrasos y la capacidad directiva o aglutinadora de cada disciplina. Se trata de una orientación dominada por el deseo de lograr una unificación de las ciencias a partir no de sus caracteres intrínsecos, sino de las redes de relaciones que tejen entre ellas en el campo de los paradigmas y los métodos; lo cual representa, en gran medida, una respuesta crítica y reformadora de un ambiente caracterizado por la superespecialización y la profesionalización, y, por tanto, gremialización e institucionalización, en su sentido más negativo, del trabajo científico. Evidentemente, el conocimiento de los resultados de estas investigaciones es de gran utilidad tanto para la construcción y mantenimiento de clasificaciones facetadas especializadas y de tesauros, como para el diseño de sistemas de representación y organización más adecuados que los actuales para la gestión de documentación de interés interdisciplinar.

\section{De la clasificación de los objetos, las ideas y los saberes a la cla- sificación de los conocimientos}

Este excurso histórico nos descubre y ayuda a valorar cuan positiva fue la aparición de la Documentación a finales del siglo XIX, para avanzar en la unificación del conocimiento científico en un período caracterizado por una división de la ciencia, casi irreparable, entre ciencias culturales y físico-naturales, con un

Scire. $1: 2$ (jul.-dic. 1995). 
matiz de superioridad añadido a estas últimas. Porque la pretensión de las primeras clasificaciones universales de reunir e integrar todas las disciplinas científicas en un mismo esquema, supuso considerar en un plano de igualdad todas las produciones del conocimiento humano. La Documentación planteaba en sus albores un arquetipo de organización del conocimiento, tal vez hoy abandonado, que en lugar de priorizar unas parcelas del saber o de la acción humana sobre otras, obedecía, exclusivamente, a un criterio funcional: organizar mejor la información que contienen los documentos para su conservación y posterior recuperación y transmisión, atendiendo tanto a su contenido como a los diferentes perfiles que poseen sus potenciales usuarios.

El espectacular y veloz crecimiento del conocimiento humano desde el siglo XVIII, planteó durante el siglo XIX, de modo paralelo al problema de cómo clasificar la realidad estudiada y las parcelas del conocimiento que se ocupan de ella, el reto de cómo proceder a la organización de las innumerables informaciones en las que se traduce la actividad cognitiva de la humanidad, de una forma capaz de garantizar su conservación y transmisión. Esta explosión de la información originó el nacimiento de las grandes bibliotecas nacionales y universitarias, de los monumentales catálogos bibliográficos y de las primeras revistas de resúmenes, y, por tanto, fomentó el desarrollo y universalización de técnicas de gestión de los documentos cada vez más precisas y eficaces. A partir de las cuales, se erigió una nueva disciplina para optimizar sus resultados: la Documentación, y nació una nuevo profesional, el gestor de la información - por encima de las diferentes especies concurrentes en su aparición-, destinado a satisfacer la creciente demanda de información por parte de los científicos en particular y de la sociedad en general.

Pero la plena comprensión del origen de esta nueva tecnología, exige enmarcar esa razón de índole "cientifista" en un contexto histórico más amplio, caracterizado por un proceso ininterrumpido de modernización social que, auspiciado por una serie continua de innovaciones tecnológicas, provocó a mediados del siglo XIX el nacimiento de la actual sociedad de la información en las naciones occidentales más avanzadas, desde donde se ha expandido a lo largo de nuestro siglo hasta todos los rincones del planeta. La sociedad de la información se caracteriza, entre otros rasgos, por una universalización y un aumento de la especialización selectiva de la demanda de información, basadas en una progresiva democratización de la generación y acceso al conocimiento, a la que no es extraña el desarrollo de unas sociedades industriales, dotadas de un organización cada vez más democrática. La democracia ha convertido en un deber y en un derecho irrenunciable la necesidad de estar informado en una infinita variedad de materias, al imponer el triunfo de la meritocracia y de la capacidad sobre el derecho de cuna como criterio de estratificación social, y despojar al hombre de la condición de

Scire. $1: 2$ (jul.-dic. 1995). 
súbdito de cualquier forma de poder, para elevarlo a la categoría de ciudadano activo, responsable, comprometido y protagonista de la acción social. La adopción de las decisiones más adecuadas para garantizar el éxito de cualquier empresa y el ejercicio de los derechos individuales del modo más conveniente para la conquista de la felicidad, exigen al hombre moderno disponer de unos criterios de actuación basados en un conocimiento preciso de la realidad sobre la que actúa y tiene derecho a modelar, que sólo la información, en cuanto que se trata de conocimiento comunicado y socializado, le puede dar. En la democracia industrial, la información es poder, ya que sólo ésta permite conocer el mundo y disponer, por tanto, de la llave que garantiza su control.

De acuerdo con esta realidad, las Ciencias de la Documentación se nos presentan ante todo como un proceso adaptativo de la humanidad, ante el reto que plantea la creciente complejidad de la sociedad moderna y su subsiguiente y proporcional producción de conocimiento y demanda de información, potenciadas a su vez por el desarrollo de las nuevas tecnologías. Se trata de un proceso dirigido a optimizar la comunicación del conocimiento, mediando entre los productores de información y sus potenciales receptores, mediante el desarrollo de una serie de técnicas, hoy consolidadas, que liberan a estos últimos de buena parte de las tareas de selección, análisis, almacenamiento y recuperación de la información, permitiéndoles la concentración en su función prioritaria: la conversión de la información relevante en conocimiento. Fruto del trabajo del profesional encargado de esas labores, el documentalista, ha aparecido una nueva realidad: la circulación de la información documental; más comúnmente conocida como el ciclo de la información, ya que se caracteriza por ser un proceso de retroalimentación donde los conocimientos son transformados en información disponible y a su vez esa información disponible condiciona la producción de nuevos conocimientos.

Las tareas conducentes al análisis del contenido de los documentos ocupan un lugar central en las fases de tratamiento y recuperación de ese ciclo de la información, ya que el éxito de la conservación y difusión del conocimiento que contienen tanto un documento como un fondo documental, dependen de su correcta representación, clasificación y puesta en relación con el presente en el resto de los documentos, de acuerdo a unos criterios y reglas precisos. Pero la eficacia de estas actividades sólo es posible gracias al desarrollo de unos efectivos y complejos instrumentos auxiliares, que han acabado convirtiéndose en el recurso fundamental e indispensable de toda empresa de representación y organización: los lenguajes documentales. De ahí, que el diseño de estas herramientas y la reflexión sobre los fundamentos de su estructura y del proceso al que sirven, hayan sido, tradicionalmente, unos de los principales motores propulsores del avance de las Ciencias de la Documentación. Así, no sorprende que sus fundadores ya se

Scire. $1: 2$ (jul.-dic. 1995). 
plantearan buena parte de su quehacer como un trabajo de taxónomos, pues, como escribían Paul Otlet y Henri Lafontaine en un folleto de presentación de la Oficina Internacional de Bibliografía Sociológica en 1894: «Porque los métodos de observación y cooperación han producido incesante progreso y la internacionalización de la ciencia ha ampliado el campo de acción de los investigadores, una clasificación rigurosamente científica ha llegado a convertirse en cosa necesaria» (cfr. López Yepes, 1989, p. 34). En este sentido, la publicación en 1876 de los dos primeros lenguajes documentales modernos: la Clasificación Decimal de Melvil Dewey y las Reglas para un Catálogo-Diccionario de Charles Ami Cutter, convierten a ese año en una de las fechas claves del nacimiento de esta nueva disciplina científica.

Sin lugar a dudas, la aparición en 1876 de las dos obras citadas, supuso una revolución sin precedentes tanto en el ámbito de la gestión de los documentos como en la esfera de la clasificación, desde la formación de los primeros depósitos documentales y los trabajos de Aristóteles, pues no sólo consolidaba y provocaba un espectacular avance de un proceso iniciado con las primeras bibliotecas y archivos de la humanidad, dotando a sus responsables de unos instrumentos de representación y organización más eficaces que los usados hasta entonces, sino que colocaba a la vanguardia de la actividad clasificadora a una nueva y revolucionaria dimensión de ésta. La labor de clasificar objetos, ideas y saberes se completaba, definitivamente, con la alternativa de organizar los documentos que describen las cosas y los conceptos, a partir de la representación y clasificación de los conocimientos que contienen, mediante unos sistemas auxiliares que se presentan de aplicación universal. Evidentemente, el objetivo de las diversas clasificaciones filosófico-científicas propuestas por diversos pensadores a lo largo de la historia, nunca había sido el mismo que persiguen las documentales, a pesar de que los primeros bibliotecarios y bibliógrafos utilizaron algunas de esas clasificaciones, tras las convenientes correcciones, para organizar los documentos, o, al menos, se inspiraron en ellas para desarrollar sistemas propios, que, de todos modos, nunca se mostraron suficientemente eficaces. Tras varios siglos de ensayos, el nacimiento de los primeros lenguajes documentales supuso el descubrimiento de un nuevo continente en el mundo de la clasificación, caracterizado por el paso de la clasificación de la realidad y del saber a la organización del conocimiento.

Obedece a algo más que a una casualidad, la aparición simultánea de dos obras que con el transcurso del tiempo se han convertido en los primeros exponentes de los dos principales tipos de lenguajes documentales: los de estructura jerárquica, que auxilian la identificación de la materia de una obra para proceder a su identificación mediante su integración en una clase dentro de una jerarquía; y los de estructura combinatoria, que permiten la organización de un fondo docu-

Scire. $1: 2$ (jul.-dic. 1995). 
mental a partir del control morfológico y conceptual y la asociación por proximidad semántica de los términos identificadores de la materia, sin necesidad de recurrir a su inclusión en una jerarquía. Ambos léxicos documentales son el resultado de la evolución de las principales soluciones que durante siglos se habían buscado a los problemas de instalación e inventario de los fondos documentales.

La instalación se basaba, habitualmente, en el uso de uno de los dos sistemas siguientes. La ordenación alfabética de los documentos según alguna dimensión unívoca, tales como el título, el autor, la materia o la tipología documental. O su ordenación temática en virtud de un esquema previo, generalmente de estructura piramidal, que podía obedecer a criterios tan dispares como el contenido o la forma física y el tamaño de los documentos, la procedencia geográfica y temporal de los autores o la institución u oficina productora. Paralelamente, se fue perfeccionando un sistema de acceso al contenido de los documentos más relevantes, consistente en la construcción de índices analíticos alfabetizados, que aunque resultaban inútiles para colocar los documentos en el depósito, enriquecían la información disponible sobre el fondo. Con el transcurso del tiempo y las consiguientes variaciones y perfeccionamiento de estos sistemas, a partir del análisis de su inadecuación para gestionar fondos en progresivo crecimiento y de las insatisfacciones que ocasionaban durante la búsqueda de información, la primera solución dió lugar a la idea y los actuales programas de control bibliográfico universal fundados sobre la normalización de la descripción, la segunda generó las clasificaciones jerárquicas bibliográficas y archivísticas y la tercera se encuentra en el origen tanto de los lenguajes documentales de estructura asociativa como de los instrumentos de descripción de archivos.

La entusiasta acogida por los especialistas norteamericanos de la publicación anónima de A Classification and Subject Index for Cataloguing and Arranging the Books and Pamphelts of Library por el joven bibliotecario niuyorkino Melvil Dewey en 1876, animó a su creador a presentar en 1885 una segunda versión ampliada, bajo el nombre definitivo de Decimal Classification, en alusión al uso del sistema decimal para la división de las clases en subsclases y para la notación de los documentos. En 1893, el también bibliotecario estadounidense Charles Cutter presentó la Expansive Classification, que a partir del principio de que una clasificación debe adaptarse al volumen del fondo de una biblioteca, contiene siete esquemas diferentes pero dependientes unos de otros, que van desde el más general al más preciso, de modo que cuando se pasa de uno a otro, basta con completar las rúbricas del índice con una notación más específica, que es de tipo alfabético. A diferencia de la Clasificación Dewey, esta clasificación no tuvo un gran éxito, pero sirvió de punto de partida para construir a finales del siglo XIX la Clasificación de la Biblioteca del Congreso, que, junto con la primera, es consi-

Scire. $1: 2$ (jul.-dic. 1995). 
derada de referencia en los Estados Unidos de Norteamérica. Durante esa misma época, los trabajos de Paul Otlet y Henri La Fontaine a partir de la Clasificación Dewey en el Instituto Internacional de Bibliografía, dieron lugar a la Clasificación Decimal Universal, cuyo uso se difundió con gran rapidez por toda Europa durante el primer cuarto del siglo XX. Desde entonces, estos sistemas de clasificación jerárquicos no han dejado de crecer, para adptarse al aumento de la variedad del conocimiento generado por el hombre, animados por su intento de servir como instrumentos que propicien el control bibliográfico universal y la difusión e intercambio mundial de información. Así, de los 932 conceptos que contenía la primera edición de la C.D.U., se ha pasado a los más de 160.000 de su cifra actual, organizados por un grupo de trabajo especial de la Federación Internacional de Documentación.

Todas estas clasificaciones parten de unos principios teóricos, en general de base filosófica e influenciados por los sistemas de las ciencias operantes en el entorno de sus autores, a partir de los cuales se genera la tabla o plan de la clasificación. La estrategia es dividir y agrupar las disciplinas científicas, las artes y las técnicas en grandes clases y subclases, en las que se incluyen primero sus dominios y luego los objetos y los productos del saber humano propios de éstos, en grupos de crecimiento indefinido progresivamente más cerrados y específicos, guiados por unos criterios de creación variables, estableciendo entre ellos una relación jerárquica basada en el principio de inclusividad de las clases. Cada clase recibe una denominación en lenguaje natural, pero se codifica con un sistema de notación, que puede ser indistintamente de tipo alfabético, numérico o alfanumérico, de acuerdo con su posición en el plan de clasificación; permitiéndose el acceso no conceptual mediante un índice alfabético. El uso de una clasificación de este tipo asegura en un centro de información tanto el almacenamiento de los documentos como su acceso por temas con la máxima especificidad requerida, ya que el código de clasificación sintético que se asigna para representar la materia de la que trata un documento, sirve al mismo tiempo para individualizarlo y ponerlo en relación con el resto en el catálogo sistemático de materias, que reproduce la estructura de la clasificación, y para permitir una instalación del fondo a partir de las clases principales, que se recoge en el catálogo topográfico sistemático.

Pero debido a que estas clasificaciones exigen del usuario un profundo conocimiento de su estructura para poder sacar durante la recuperación todo el partido que ofrecen, y que el uso de las tablas y los índices se reserva generalmente a los especialistas, los primeros bibliotecarios modernos ya se vieron obligados a completar el catálogo sistemático de materias con un instrumento de búsqueda de información más simple: el catálogo alfabético de materias, que acompaña al catálogo alfabético de autores y títulos. Este catálogo recuerda a un diccionario

Scire. $1: 2$ (jul.-dic. 1995). 
por el tipo de sus entradas y por su estructura, de modo que la recuperación resulta fácil e intuitiva. Sin embargo, para evitar la ambigüedad y la incertidumbre que el uso del lenguaje natural presenta durante la formulación de preguntas, se necesita un instrumento que garantice el control morfológico y semántico del vocabulario usado: una lista de autoridades. Este lenguaje documental presenta una serie de reglas simples para la construcción y ordenación de encabezamientos de materia a partir de una lista de términos autorizados para su uso dentro de un sistema documental específico o para un ámbito determinado del conocimiento, que completan la representación del contenido de un documento otorgada por la notación extraída de un sistema de clasificación. Las primeras listas de autoridades fueron de creación local, pero no tardaron en aparecer grandes modelos destinados a sustituir o inspirar a esos lenguajes propios de cada biblioteca, como la Lista de Encabezamientos de Materia de la Biblioteca del Congreso de Washington, la Lista de Encabezamientos de Materias de M. E. Sears o, más recientemente, diversas listas nacionales.

La aparición y desarrollo de este tipo de lenguaje documental en el mismo momento que las clasificaciones documentales, no es pues un producto del azar, sino que, evidentemente, es el resultado tanto de la evolución y maduración de la antigua técnica bibliotecaria de construcción de índices analíticos, como de la obligación de responder a una necesidad planteada por el uso de esas clasificaciones si se quiere garantizar una recuperación más sencilla, eficaz y precisa de los documentos. Sin embargo, a pesar de que la introducción del principio de entrada directa compite con el esquema arbóreo de las clasificaciones documentales, su aparición no supuso ningún cambio importante en el modo de análisis del contenido de los documentos. Su uso sigue basándose en la identificación del tema principal y los secundarios, si ha lugar, presentes en un documento, sin profundizar en el descubrimiento y representación de todas las unidades de información relevantes y pertinentes que éste contiene.

Ese cambio de orientación hubo de esperar hasta mediados del siglo XX, gracias a la coincidencia en el tiempo de dos factores. En primer lugar, la formulación y difusión de las críticas por diversos autores, a partir de la reflexión de S. R. Ranganathan, a los límites que presenta el uso de sistemas de clasificación jerárquicos y de encabezamientos de materia en los procesos de representación y organización, por basarse en la formulación global del contenido de un documento mediante una materia, en lugar de por el conjunto de conceptos que construyen tanto un documento como una materia. La reivindicación de la representación de un documento a partir de sus nociones más elementales pero dotadas de significado relevante para el sistema de información, hizo más evidente la presencia de defectos como la rigidez estructural, la insuficiencia de las relaciones semánticas, la incapacidad para mostrar una materia desde diferentes perspecti-

Scire. $1: 2$ (jul.-dic. 1995). 
vas y el aspecto poco amigable, si no esotérico, de la notación, presentes en las clasificaciones jerárquicas desde su nacimiento. $\mathrm{Y}$, el segundo factor concurrente en ese cambio de perspectiva, consiste en el desarrollo de lenguajes de indización como las listas de palabras claves y de descriptores, auspiciados por los avances en las técnicas de acumulación de datos en archivos informáticos y la aparición de los primeros programas de indización automática y de búsqueda en lenguaje natural. La necesidad de sacar partido de las ventajas que ofrece para el acceso a la información, la combinación de la velocidad de las técnicas automatizadas de búsqueda y la posibilidad de usar el lenguaje natural, ha favorecido la creación y proliferación de instrumentos de control de vocabulario que permitan el uso de técnicas como el truncamiento y el enmascaramiento de los términos de interrogación y su combinación mediante los principios de la lógica booleana.

Finalmente, la conjunción de los principios de representación y organización presentes en los sistemas de clasificación facetados, con el desarrollo de la tecnología informática y el perfeccionamiento de las técnicas de estructuración y asociación de conceptos implíticos en las clasificaciones jerárquicas y los lenguajes de indización, respectivamente, ha propiciado el nacimiento y expansión en las últimas tres décadas de la más precisa herramienta de control terminológico y, por tanto, documental, construida hasta el presente: el tesauro. Este lenguaje documental supera a las listas de autoridades, al aumentar las posibilidades en la precisión de los términos de indización tanto durante el tratamiento como durante la búsqueda de información, gracias a que añade las relaciones jerárquicas y asociativas a la de equivalencia y a que permite la coordinación de los términos de indización durante la fase de recuperación. Y supera a las clasificaciones jerárquicas, en cuanto a que sin desperdiciar las posibilidades implícitas en la inclusión de clases para favorecer la precisión del contenido conceptual de cada descriptor y guiar el hallazgo del término más específico para representar un concepto, enriquece la definición del concepto y la estrategia de asignación de descriptores mediante la incorporación de relaciones de equivalencia y asociativas.

\section{Novedades y pervivencias de las primeras clasificaciones docu- mentales}

La plena comprensión del significado de los lenguajes documentales en la historia de la clasificación humana, exige efectuar una comparación de los elementos y los rasgos estructurales de las primeras clasificaciones bibliográficas universales y jerárquicas con los sistemas de las ciencias de su época. Asimismo, el análisis de las semejanzas y las diferencias entre ambos, nos permitirá valorar en su justa medida las novedades que presentan ese tipo de lenguajes documentales pero también descubrir algunos de sus caracteres como huellas del entorno en el que surgieron. Innovación y continuismo se hallan presentes en las prime-

Scire. $1: 2$ (jul.-dic. 1995). 
ras clasificaciones documentales bajo la forma de una simbiosis de rasgos rupturistas y de pervivencias respecto a los sistemas de clasificación contemporáneos.

Las diferencias más relevantes entre las clasificaciones documentales y las clasificaciones filosóficas y científicas son fundamentalmente las siguientes. Los autores de las primeras se interesan por la dimensión práctica de la actividad clasificatoria, midiendo su nivel mediante un criterio utilitarista; mientras que los creadores de las segundas se preocupan, generalmente, más por los aspectos teóricos y especulativos que rodean a la clasificación. Por eso, a menudo, el único motivo de existencia de una clasificación de las ciencias es satisfacer una inquietud intelectual de su autor, frente a las clasificaciones documentales, que se generan para instalar documentos en un fondo y satisfacer las demandas de los usuarios de los centros de información. La ausencia de una función práctica genera unas clasificaciones de las ciencias habitualmente poco detalladas y extensas, mientras que su presencia en las clasificaciones documentales provoca una minuciosa subdivisión de cada materia, donde los elementos se agrupan de acuerdo con las mutuas relaciones que establecen entre ellos. De ahí que los teóricos de la ciencia se limiten a agrupar ideas abstractas en clases de acuerdo con el principio de filiación, pero los constructores de las clasificaciones documentales se dediquen a establecer relaciones entre conceptos, sobre todo de género especie y partitivas. De acuerdo con lo anterior, los sistemas de las ciencias no necesitan ni introducir clases generales y de forma ni de la presencia de un índice que facilite su acceso; en cambio, las clasificaciones documentales exigen clases generales que permitan acomodar a los documentos cuyo contenido concierne a muchas parcelas del universo de las ideas, clases de forma que puedan actuar como axuliares del número de clasificación principal asignado a un documento y la existencia de un índice alfabético de materias que facilite el acceso. Esa vocación pragmática de las clasificaciones documentales origina la necesidad de otorgar a cada materia un número de clasificación peculiar, según las convenciones de un sistema numérico ordinal, que al tiempo que la singulariza del resto representa el grado de relación que mantiene con el resto de las materias; lo cual exige, en consecuencia, la previsión de normas para asignar un número de clasificación a cada nueva materia que pueda incorporarse al sistema, ya que su éxito depende de su capacidad para adaptarse a los cambios y las ampliaciones exigidas por el constante crecimiento del conocimiento humano. De la suma de todas esas diferencias resulta que las clasificaciones de las ciencias presentan un acceso sencillo y simple, frente a la difícil y compleja accesibilidad de las bibliográficas, debido, sobre todo, a las limitaciones impuestas por las convenciones de notación y la necesidad de proceder a su mantenimiento. Como consecuencia de esa complejidad, el autor de una clasificación documental debe acompañarla de la exposición de los principios en que basa su estructura, las reglas que rigen su uso y el modo de inte-

Scire. $1: 2$ (jul.-dic. 1995). 
grar la expansión del universo de las ideas. En cambio, el creador de una clasificación de las ciencias no tiene la necesidad de presentar una teoría de la clasificación explicativa del porqué de su estructura (Bhattacharyya y Ranganathan, 1974, p. 119-143).

Sin embargo, pese a estas diferencias, las clasificaciones bibliográficas universales pueden considerarse, en un sentido amplio, un tipo de clasificaciones filosóficas y científicas, en cuanto que tratan de agrupar y relacionar dentro de clases las diversas disciplinas que se ocupan del conocimiento del mundo. Mas se trata de un tipo bien singular, ya que esa serie de novedades que presentan, generan tres rasgos de su carácter que las diferencian con amplitud del resto de sus compañeras: una vocación pragmática y funcional, una elevada potencia clasificatoria y una profunda mejora estructural. Un único objetivo guía a los constructores de las clasificaciones documentales: crear una herramienta capaz de servir para proceder a la organización del conocimiento producido por los pensadores y científicos, de un modo a la vez sencillo y efectivo. En función de lo cual poseen una amplitud y una tendencia expansiva en el número de clases, que les permite cubrir y contener el universo de las ideas con mucho más detalle que los sistemas de las ciencias, hasta el punto de que consisten más en una clasificación de los productos del saber y de la acción humana, que de las disciplinas y subdisciplinas en que se dividen ese saber y esa acción. Participando, además, para satisfacer ese objetivo de servicio, de una estructura enriquecida con ajustes tales como la introducción de clases generales y de forma, la presencia de un sistema numérico destinado a asignar un número de clasificación singular a cada materia, que a su vez se otorgará a los documentos clasificados, y la definición de una serie de normas que preveen la introducción de nuevas materias.

La coincidencia de estos tres rasgos en las clasificaciones bibliográficas universales provocó a finales del siglo XIX un salto cualitativo en la historia de la clasificación, pues se mostraron bastante más eficaces que otras herramientas más cercanas a los caracteres estructurales de los sistemas de las ciencias para proceder a la organización de los documentos científicos, técnicos y literarios impresos, con vistas a su conservación y transmisión. Sin embargo, no por eso dejan de tener deudas con el contexto clasificatorio en el que surgieron; es decir, también presentan ciertas semejanzas con las clasificaciones de las ciencias y las taxonomías operantes en la época de su nacimiento.

Evidentemente, el rasgo más característico, y también el más criticado en la actualidad, de los primeros sistemas de organización documental del conocimiento, es su rígida estructura jerárquica, que provoca la consiguiente compartimentación — no confundir con división — del saber. Pero la comprensión de por qué los primeros lenguajes documentales eligieron una organización jerárquica de los elementos y clases que la componen, que favorece el aislamiento y la inco-

Scire. $1: 2$ (jul.-dic. 1995). 
municación entre las disciplinas y los objetos del conocimiento dentro del sistema de clasificación, no puede explicarse únicamente por la evolución de una tradición bibliotecaria, apuntada más arriba. Esa explicación se encuentra, fundamentalmente, en otros dos hechos. En primer lugar, porque aunque la presencia de las entidades concretas y específicas sobre las que opera el conocimiento en las clases medias e inferiores, invitaban al reconocimiento de categorías de agrupación y observación que pudieran introducir unas relaciones distintas a las genéricas - como sucede en las clasificaciones facetadas-, su convivencia en un mismo sistema con la división de las ciencias y de las artes en disciplinas en las clases superiores, como era propio de las clasificaciones filosófico-científicas, dificultaba ese progreso taxonómico. Es decir, invitaba a asumir de un modo acrítico e irreflexivo la jerarquización del conocimiento común a la mayoría de las clasificaciones científicas contemporáneas, continuando una tradición tan vieja como la razón filosófica y la razón científica, como hemos observado en nuestro breve recorrido por la historia de las clasificaciones del saber hasta finales del siglo XIX. Y, en segundo lugar, también influyeron las características que adoptaba la ciencia decimonónica. Por una parte, la consolidación de la especialización científica frente a la ciencia única y la continua aparición de microdisciplinas impedía el desarrollo de perspectivas interdisciplinares e integradoras del pensamiento científico, provocando la incomunicación de las ciencias, cuya unión sólo se basaba en la elevación del método experimental e hipotéticodeductivo al rango de método científico por antonomasia. Y, por otra parte, el hecho de que todos los avances en el conocimiento de la realidad física y cultural se presentaran desde el siglo XVIII bajo la forma de taxonomías de estructura jerárquica, siguiendo el modelo impuesto por las ciencias naturales, con independencia de la adopción de un criterio de construcción de las clases basado en las semajanzas y diferencias de forma o en la evolución histórica de los elementos componentes.

Las marcas del tiempo en las clasificaciones bibliográficas universales son de tal envergadura, que no sólo las encontramos en su estructura y en la división del saber propuesta, sino también en sus caracteres ideológicos. El intento de presentarse como universales debe entenderse como la asunción de la utopía operante desde la Ilustración sobre la posibilidad de dominar a la Naturaleza mediante el desvelamiento de sus secretos a partir de la reducción de los fenómenos a una explicación científica racional y, por tanto, válida para toda época y lugar. En este sentido, la jerarquización es el medio que mejor permite apropiarse de la realidad si se elabora una imagen previa de ésta como un mundo racional y perfecto, en cuanto que la conversión de cada uno de sus elementos en un taxón dentro de un sistema perfectamente organizado, es la máxima expresión de que el sujeto científico se ha apropiado de su objeto de conocimiento mediante el conve-

Scire. $1: 2$ (jul.-dic. 1995). 
niente proceso de reducción de sus caracteres y limitación de sus relaciones. La complejidad del Universo no es entendida como una tensión inestable de fuerzas en un proceso continuo de cambios en las relaciones entre sus elementos, que provocan incluso variaciones en la naturaleza de sus elementos, que abren el paso a nuevos tipos de relaciones cada vez más y más complejas. En el pensamiento científico del siglo XIX dominaba la idea de orden entendido como simplificación y aislamiento en lugar de como relación y complejidad, donde no hay sitio ni para la riqueza relacional entre elementos pertenecientes a diversas clases ni para el azar ni para la aberración o lo que está fuera de la norma. De ahí el mito del progreso continuo en el avance del conocimiento, el reduccionismo de la riqueza de la realidad, la desintegración de la unidad del saber, la negación de las excepciones al esquema de comprensión construido por una razón que se pretende universal y, por tanto, superior a las razones elaboradas por otras culturas, sin caer en la cuenta de su carácter eurocéntrico. Una serie de caracteres que asumen plenamente las primeras clasificaciones documentales, como buenas hijas de su tiempo.

Pero quizá la más importante y trascendental pervivencia de las clasificaciones filosófico-científicas en las documentales, se encuentra en el mantenimiento durante su construcción de una aproximación subjetiva a la realidad a clasificar, pese a que las clasificaciones especializadas y las sucesivas ediciones de los principales sistemas universales se han centrado más en las entidades concretas y específicas del conocimiento, que en la división de las ciencias y de las artes en disciplinas. Tal vez este hecho es inevitable, porque del mismo modo que toda actividad científica tiene unos fundamentos teóricos y obedece a unos intereses determinados — pues incluso la investigación más empírica se encuentra guiada por una idea previa de lo que se quiere no sólo buscar, sino también encontrar, bajo toda clasificación documental subyace una concepción del mundo y una valoración, más o menos consciente, acerca de los criterios más válidos para proceder a la división y distribución en clases del conocimiento que se posee de la realidad. No obstante, el reconocimiento de la existencia de un componente subjetivo en toda clasificación documental, no debe entenderse como una negación de la posibilidad de clasificar, como hemos demostrado en la anterior colaboración.

\section{Conclusión}

Por encima de estas diferencias y semejanzas entre los rasgos de las clasificaciones documentales con otros tipos de clasificaciones, es evidente que existe una distinción sustancial en los elementos que clasifican y los objetivos que persiguen. El científico se preocupa de organizar la comprensión de los fenómenos de la naturaleza física y social que investiga, generando taxonomías a partir de un

Scire. $1: 2$ (jul.-dic. 1995). 
proceso analítico que depende estrechamente del método de observación que adopta. El filósofo de la ciencia se interesa por organizar las disciplinas del saber y desvelar sus relaciones, movido por el interés de descubrir el estado en el que se encuentra la ciencia y de perfeccionar el método científico. Y, el constructor de sistemas de representación y organización documental se dedica a construir y mantener herramientas capaces de conservar del modo más eficaz posible, desde la perspectiva de la recuperación para la transmisión, el conocimiento acumulado en los documentos, garantizando de este modo su conversión en información capaz de generar nuevo conocimiento.

En definitiva, la aparición de los taxónomos documentales provocó la apertura de un nuevo horizonte en la disciplina de la Taxonomía: la clasificación del conocimiento humano fijado en los documentos. Pero el nacimiento de esta nueva actividad no supuso ni la desaparición ni la ignorancia de los sistemas de clasificación de las ciencias y de la naturaleza. Esos trabajos taxonómicos todavía gozan de una gran vitalidad. Y los ingenieros del conocimiento deben saber aprovecharse de sus depurados métodos y de sus resultados en el hallazgo de las relaciones entre las ciencias y entre los objetos de la realidad, para mejorar la operatividad y la actualidad de los lenguajes documentales, al igual que hicieron los padres de las primeras clasificaciones bibliográficas.

\section{Referencias}

Apostel, L. ; Kiriakoff, S. G. et. al. La classification dans les sciences. París, 1963.

Bhattacharyya, G., Ranganathan, S. R. From knowledge classification to library classification. // Wojciechowski, J. A. (ed.). Conceptual basis of classification of knowledge. Münich : Verlag Dokumentation, 1974. p. 119-143.

Bliss, H. E. The organization of knowledege and the system of the sciences. New York : Wilson \& Co., 1929.

Cassirer, E. El problema del conocimiento. México : Fondo de Cultura Económica, 1948. 4 vols.

Curras, E. The vertical integration of science as a stabilizing Factor for Mankind. // Rodriguez, R. ; Banathy, B. H. International Systems Science Handbook. Samos : University of Aegean, 1992. p. 3.7.1-3.7.17.

De Grolier, E. Étude sur les catégories générales applicables aux classifications et codifications documentaires. París : Unesco, 1962.

De Grolier, E. Théorie et pratique des classifications documentaires. París : UFOD, 1956

Dobrowolski, Z. Étude sur la construction des systèmes de classification. Paris : GauthierVillars, 1964.

Garcia Marco, F. J. ; Esteban Navarro, M.A. On some contributions of the Cognitive Sciences and Epistemology to a Theory of Classification. // Knowledge Organization. $20: 3$ (1993) 126-132. 
Garcia Marco, F. J. Principios del análisis y representación del contenido. // Rubio, Emilio (ed.). Documentación Médica. Zaragoza : Universidad de Zaragoza, 1992.

Kumar, K. Theory of Classification. 4. ${ }^{\text {a }}$ ed. Nueva Delhi : Vikas Publishing, 1988.

López Yepes, J. Paul Otlet y la fundamentación de la Ciencia de la Documentación. // López Yepes, J. (comp.). Fundamentos de Información y Documentación. Madrid : EUDEMA, 1989, p. 33-45.

López-Huertas Pérez, M. ${ }^{\mathrm{a}}$ J. Lenguajes documentales: aproximación a la evolución histórica de un concepto. // Boletín de la Anabad. 41 : 1 (1991) 61-70.

Maniez, J. L'évolution des langages documentaires. // Documentaliste : Sciences de l'Information. $30: 4-5$ (1993) 254-259.

Norris, D. M. A history of cataloguing and cataloguing methods, 1100-1850. Londres : Grafton, 1939.

Parkhi, R. S. Library classification : evolution of dynamic theory. Nueva Delhi : Vikas Publishing, 1972.

Salvan, Paule. Esquisse de l'évolution des systèmes de classification. Paris : Ecole Nationale Supérieure des Bibliothécaires, 1967, 76 p.

Serrai, A. Le classificazioni: idee e materiali per una teoria e peruna storia. Florencia : Leo S. Olschki Editore, 1977.

Umarani, A. Knowledge Classification : A Permanent Structure for Dyamic Knowledge. // Cognitive Paradigms in Knowledge Organisation : Second International ISKO Conference, Madras 26-28 August 1992. Bangalore : Sarada Ranganathan Endowment for Library Science, 1992. p. 211-222.

Vegetti, Mario: Los orígenes de la racionalidad científica : el escapelo y la pluma. Barcelona : Península, 1981.

Vickery, B. C. Classification and indexing in science. 3rd. ed. Londres : Butterworths Scientific Publications, 1975.

Wojciechowski, J.A. (ed.). Conceptual basis of classification of knowledge. Münich : Verlag Dokumentation, 1974.

Scire. 1 : 2 (jul.-dic. 1995). 